\section{JTI}

JOURNAL OF

TRAUMA AND INJURY

Received: June 2, 2020

Revised: August 12, 2020

Accepted: August 31, 2020

\section{Correspondence to}

Jun-Min Cho, M.D.

Division of Trauma Surgery, Department of General Surgery, Korea University Guro Hospital, 148 Gurodong-ro, Guro-gu, Seoul 08308, Korea

Tel: +82-2-2626-1114

Fax: $+82-2-2626-1148$

E-mail:nobleedings@gmail.com

\title{
Hemorrhagic Shock in a Patient with a Morel-Lavallée Lesion Combined with Active Arterial Bleeding without Frac- ture
}

\author{
Eic Ju Lim, M.D. ${ }^{1}$, Jong-Keon Oh, M.D., Ph.D. ${ }^{1}$, Jae-Woo Cho, M.D., Ph.D. ${ }^{1}$, \\ Seungyeob Sakong, M.D. ${ }^{1}$, Jun-Min Cho, M.D. ${ }^{2}$ \\ ${ }^{1}$ Department of Orthopedic Surgery, Korea University Guro Hospital, Seoul, Korea \\ ${ }^{2}$ Department of General Surgery, Korea University Guro Hospital, Seoul, Korea
}

A Morel-Lavallée lesion is a closed degloving injury caused by traumatic separation of the skin and subcutaneous tissue from the underlying fascia. However, since physicians tend to focus on treating the bone fracture, hemodynamic instability accompanying a Morel-Lavallée lesion can sometimes be overlooked. We report the case of a hemodynamically unstable 73-year-old man who had a Morel-Lavallée lesion of the thigh, but no femur fracture. Angiography showed active bleeding from the muscular branch of the right deep femoral artery, which was then successfully embolized.

Keywords: Morel-Lavallee lesion; Hemodynamic instability; Embolization

\section{INTRODUCTION}

A Morel-Lavallée lesion is a closed degloving injury caused by the traumatic separation of the skin and subcutaneous tissue from the underlying fascia [1], mostly caused by severe trauma to the pelvis or thigh from motor vehicle accidents [2]. Because physicians tend to focus on treating the bone fracture, hemodynamic instability accompanying a Morel-Lavallée lesion, on account of its closed nature, can sometimes be overlooked [3]. As there are few descriptions in the literature of a Morel-Lavallée lesion combined with active arterial bleeding, we report the case of a hemodynamically unstable trauma patient who demonstrated a Morel-Lavallée lesion combined with (http://creativecommons.org/licenses/by-nc/4.0/) which permits unrestricted noncommercial use, distribution, and reproduction in any medium, provided the original work is properly cited. 
active bleeding in the muscular branch of the right deep femoral artery.

\section{CASE REPORT}

A 73-year-old man presented at emergency department of Korea University Guro Hospital after having his thigh trapped under the wheel of a truck. Upon arrival, his systolic blood pressure (110 $\mathrm{mmHg}$ ), heart rate ( 98 beats per minute), respiration rate (20 times per minute), and body temperature $\left(36.2^{\circ} \mathrm{C}\right)$ were recorded. He had a history of hypertension and no other significant medical conditions. On clinical examination, a reddish color change was observed from the proximal to middle right thigh (Fig. 1), but there was no apparent bony instability in the lower extremity. An initial Focused Assessment with Sonography for Trauma (FAST) examination was performed
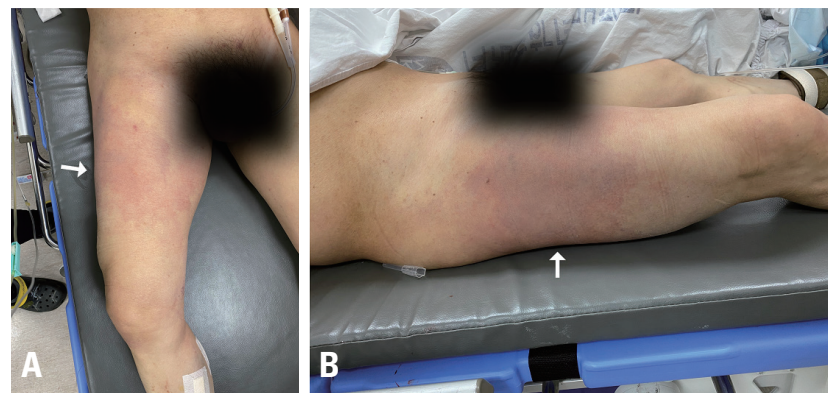

Fig. 1. (A) Anterior view and (B) lateral view of a reddish skin lesion in the right middle thigh (white arrow).

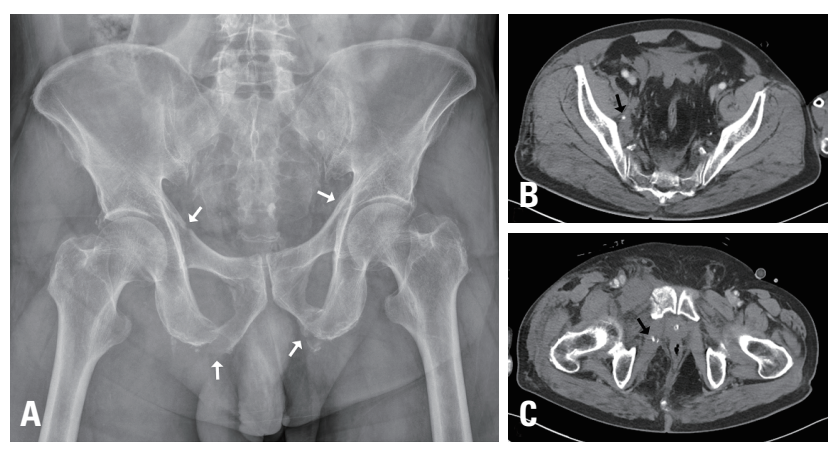

Fig. 2. (A) Pelvic anteroposterior radiographs shows a stable pelvic ring injury with bilateral superior and inferior ramus fractures (white arrows) without a posterior pelvic ring injury. An enhanced computed tomography scan demonstrates focal dot-like enhancement (black arrow) in the right (B) pelvic side wall and (C) obturator internus muscle. using a portable ultrasound machine, with negative findings.

One hour later, the patient suddenly developed mental drowsiness with dyspnea, and his systolic blood pressure dropped to $70 \mathrm{mmHg}$ and heart rate increased to 126 beats per minute. Immediate intubation was performed because of the patient's decreased level of consciousness. The first laboratory test performed 2 hours after arrival showed a hemoglobin level of $9.7 \mathrm{~g} / \mathrm{dL}$ and hematocrit of $31.8 \%$. In an immediate response to hypotension, the patient received a 1-L loading of plasma solution and three units of packed red blood cells (PRBCs). During resuscitation, a computed tomography (CT) scan was performed to evaluate the focus of the bleeding, despite the systolic pressure of $68 \mathrm{mmHg}$. After the CT scan, the patient had a systolic pressure of $72 \mathrm{mmHg}$ and received an additional two units of PRBCs and five units of fresh frozen plasma (FFP). The abdominopelvic CT scan demonstrated a pelvic ring injury that consisted of a bilateral superior and
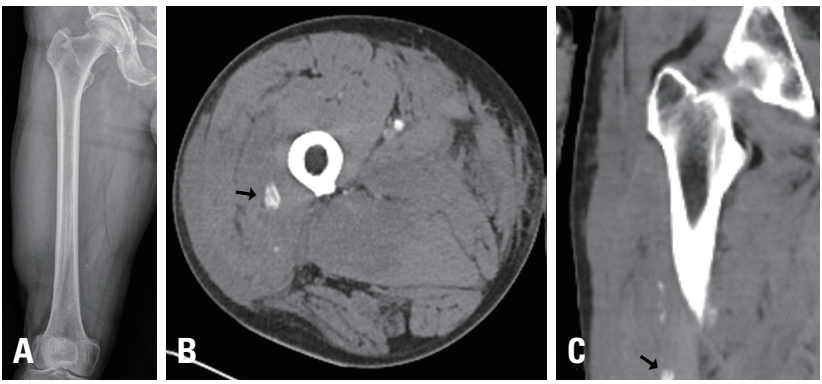

Fig. 3. (A) Femur anteroposterior radiographs present intact femur bone. An enhanced computed tomography scan demonstrates active extravasation of the muscular branch of the right deep femoral artery (black arrow) in the (B) axial view and (C) coronal view.
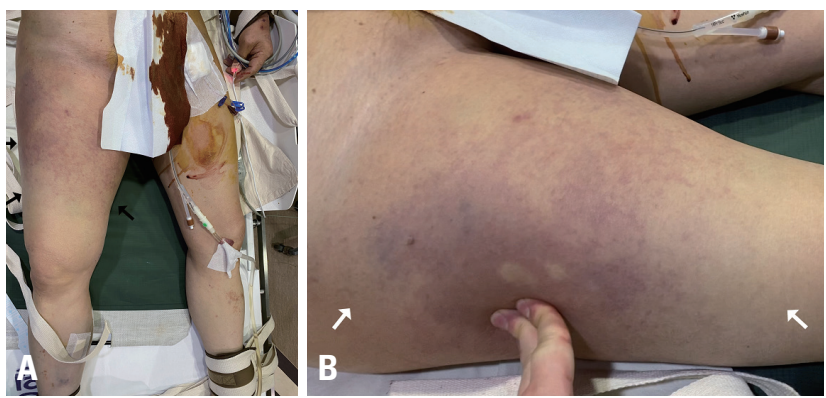

Fig. 4. (A) Huge swelling over the entire right thigh (black arrow). (B) The skin lesion became more prominent after 2 hours postinjury (white arrows). 
inferior ramus fracture without a posterior pelvic ring injury, accompanied by focal dot-like enhancement in the right pelvic side wall and obturator internus muscle (Fig. 2). The CT scan revealed extravasation of contrast in multiple instances in the thigh region without fracture of the femur (Fig. 3). There were no other injuries on brain, chest, and abdominopelvic CT scans. In addition, a
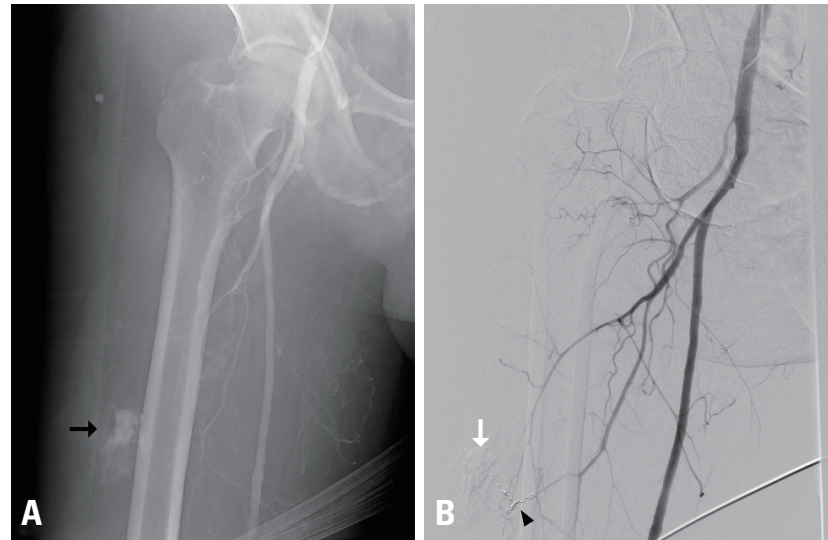

Fig. 5. (A) An angiogram demonstrates active extravasation from the muscular branch of the right deep femoral artery (black arrow) and (B) successful embolization without evidence of extravasation (white arrow) using coils (black arrowhead).

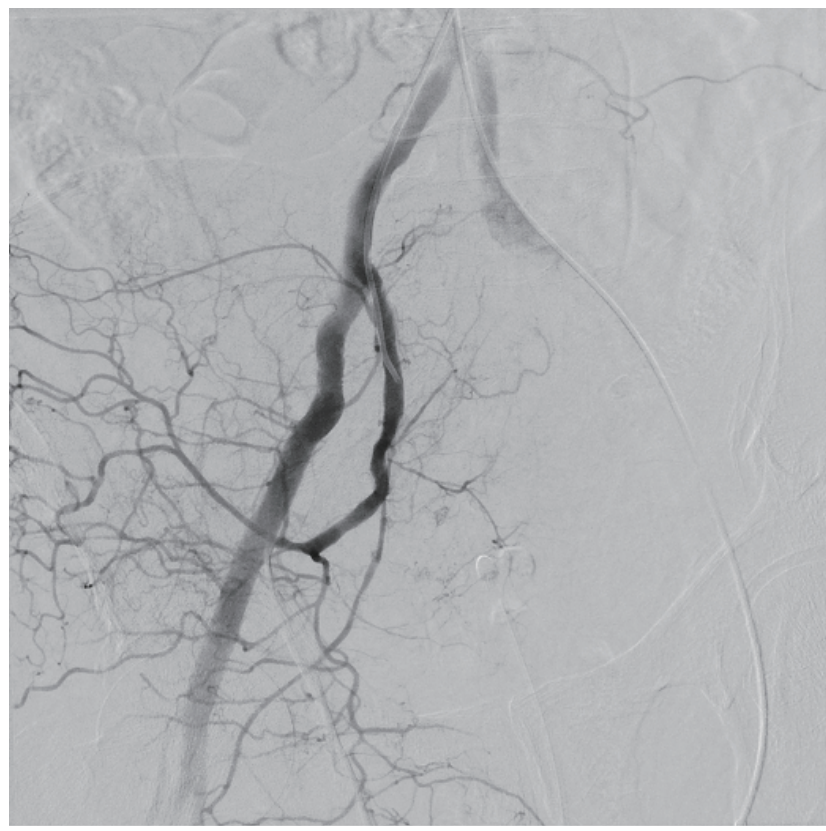

Fig. 6. A right internal iliac angiogram demonstrates no lesion correlated with the computed tomography scan in the right-side pelvic cavity. clinical examination demonstrated huge swelling over the entire right thigh. Fluctuant swelling was observed with a more apparent reddish skin lesion (Fig. 4).

Emergent embolization was performed with a coil by a radiologist to stop the bleeding, after the muscular branch of the right deep femoral artery had been identified as the source of the bleeding (Fig. 5). No lesion was correlated with the CT scan in the right-side pelvic cavity on the arteriogram (Fig. 6). After embolization, a compressive dressing was placed on the proximal thigh to limit it. The patient was admitted to the intensive care unit, where his vital signs stabilized (systolic blood pressure: $116 \mathrm{mmHg}$,

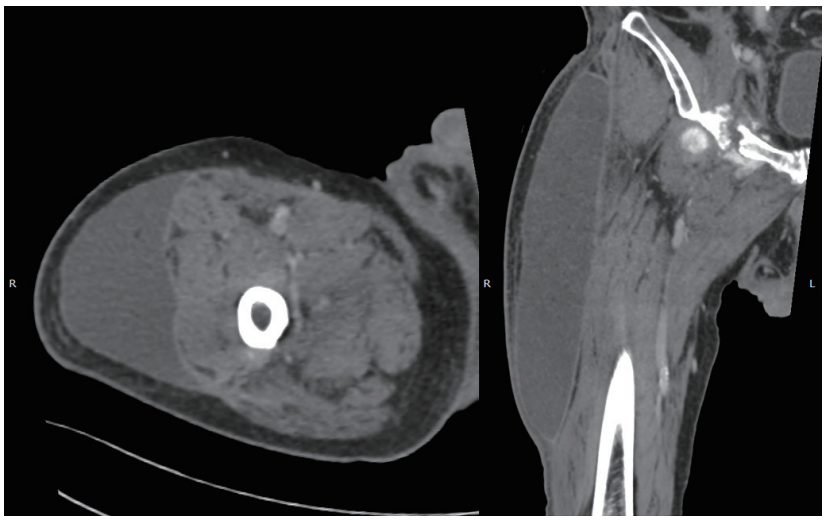

Fig. 7. An extremity computed tomography scan presents a huge amount of fluid from the right hip to the distal thigh between the subcutaneous layer and deep fascia, with a longitudinal length of $30.5 \mathrm{~cm}$.
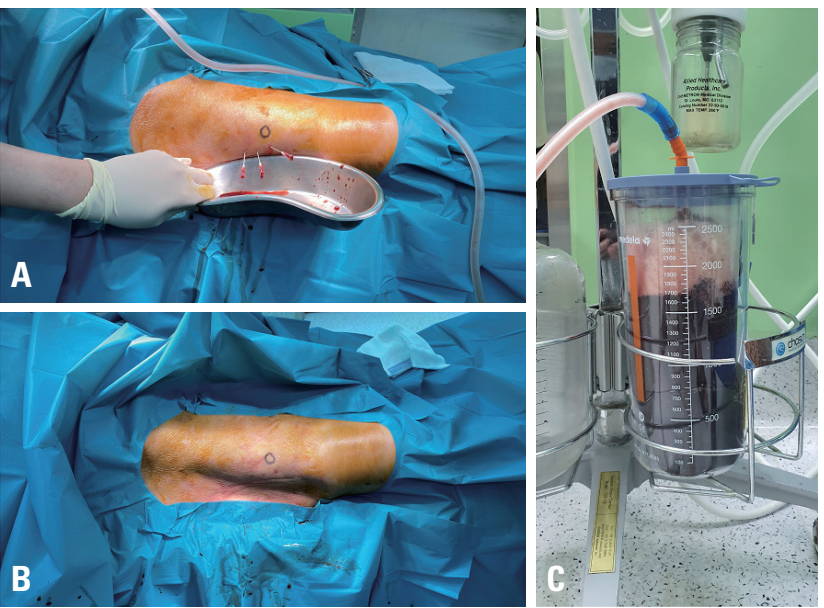

Fig. 8. (A) Percutaneous needle drainage at the middle of the lesion, where fluid has accumulated. (B) After drainage, it is possible to identify that the accumulated fluid escaped through soft tissue shrinkage. (C) Total drainage of 1,700 mL. 
heart rate after 2 hours postembolization: 95 beats per minute). He received a total of 10 units of PRBCs, 10 units of FFP, and eight platelet concentrate transfusions during the first 24 hours postinjury. He underwent extubation after 3 days and was admitted to the general ward after 5 days. Eighteen days after the injury, he had a painful swelling of the proximal thigh with palpable fluctuation and there was huge amount of fluid on a CT scan (Fig. 7). There was no evidence of a complicated fluid collection, and his body temperature did not rise above $37.8^{\circ} \mathrm{C}$ during admission, although it was maintained between $37.3^{\circ} \mathrm{C}$ and $37.8^{\circ} \mathrm{C}$. The patient underwent surgical percutaneous drainage under local anesthesia with a 16-G needle, which drained 1,700 mL of old dark bloodlike fluid (Fig. 8), and percutaneous catheter drainage was also done for 5 days. Compressive dressings were placed around the entire thigh. The result of the fluid culture test was negative. The patient completely recovered.

\section{DISCUSSION}

In this case, the clinicians did not expect hypovolemic shock, which was caused by a Morel-Lavallée lesion combined with active bleeding. The patient's early clinical presentation at the emergency department was deceptive, even if there had been an active hemorrhage in a large occupiable space. This case underscores the need for clinicians to prepare for the possibility of hypovolemic shock in patients with a Morel-Lavallée lesion, even in the absence of an unstable bony injury.

Hudson et al. [4] reported that the diagnosis of Morel-Lavallée lesion was missed in one-third of patients at the initial evaluation in 1992. Although a Morel-Lavallée lesion can lead to an accumulation of blood and lymph resulting from an injury of the rich vascular supply and lymphatics, its initial presentation can be vague [5]. The greater trochanter is the most common location of Morel-Lavallée lesions, but Morel-Lavallée lesions can still occur in the absence of a fracture, causing missed diagnoses. If the diagnosis is missed, complications such as infection, capsulation, intractable Morel-Lavallée lesion, neurovascular compromise, and cosmetic problems can occur [6].
The present case was accompanied by combined arterial bleeding from a branch of the right deep femoral artery, which has been described in previous case reports. The primary causes of deep femoral artery injuries are fracture fragments, over-penetration of a drill bit, prolonged irritation by a protruded screw, and improperly positioned retractors [7]. Potenza et al. [8] described the laceration of a branch of the deep femoral artery caused by a beak of the lesser trochanter fragment; in that patient, the hemoglobin level decreased from $9.4 \mathrm{~g} / \mathrm{dL}$ to $6.8 \mathrm{~g} / \mathrm{dL}$, even though four units of packed red cells were transfused. Mueller et al. [9] reported massive hemorrhage in a case of blunt extremity trauma without femur fracture. They recommended angiographic analysis when persistent unexplained hypotension occurs in patients with a swollen thigh. These reports imply that injuries of a single branch of the deep femoral artery can lead to a hemodynamically unstable status. In addition, bleeding is generally expected to stop in the thigh region through the help of the tamponade effect [10]. However, in the present case, the Morel-Lavallée lesion in combination with arterial bleeding resulted in hemorrhagic shock. Although it is unlikely that the volume of bleeding at the time of injury was $1,700 \mathrm{~mL}$, the volume that was extracted by percutaneous drainage, this patient is nonetheless likely to have had a massive hemorrhage in a large-volume space.

Compartment syndrome was also of concern. Nooh et al. [11] described acute thigh compartment syndrome caused by an initially undiagnosed injury of a descending branch of the lateral femoral circumflex artery. Active bleeding and large hematoma in the anterior compartment were revealed through an arteriogram, and embolization was performed. There was a report that compartment syndrome of the thigh was caused by a stable pelvic ring injury in a 94-year-old woman receiving chronic anticoagulation therapy [12]. However, in the present case, the fascia dividing compartments seem to have been disrupted by the shearing force that caused the Morel-Lavallée lesion. As a result, bleeding was not contained specifically to one compartment and the entire thigh was swollen.

In this case, the patient was hemodynamically stable upon initial clinical presentation, without a femur fracture. An hour later, with active bleeding in his thigh, the 
patient progressed to a hemodynamically unstable condition that required embolization of the active bleeding site. This implies that a clinical decision based only on a patient's initial vital signs and FAST can be dangerous if injury mechanisms are not considered. Chen et al. [13] demonstrated that prehospital vital sign trends are unlikely to be diagnostically useful because of nondirectional fluctuations. In cases of high-energy trauma, such as traffic injuries, crush injuries, and falls from a height, a clinician should be prepared for a changeable situation.

There is controversy regarding the timing and type of treatment in the management of Morel-Lavallée lesions. Regarding the timing of treatment, we delayed management for 18 days to ensure that the bleeding in the Morel-Lavallée lesion had completely stopped although embolization was performed. Another reason for the delayed management was the lack of evidence of complicated fluid collection. Several small studies reported the efficacy of conservative management, aspiration, sclerodesis, and open surgical procedures [14]. Nickerson et al. [15] presented a guideline recommending an operative intervention when more than $50 \mathrm{~mL}$ of fluid is aspirated. In this patient, however, percutaneous aspiration was performed in consideration of the facts that the patient was relatively old, skin necrosis was not present, and there was no complicated fluid collection.

In conclusion, even if a patient is stable without an apparent unstable fracture at the time of admission to the emergency department, a Morel-Lavallée lesion should be observed carefully because a huge amount of bleeding can be contained without any interference.

\section{REFERENCES}

1. Hak DJ, Olson SA, Matta JM. Diagnosis and management of closed internal degloving injuries associated with pelvic and acetabular fractures: the Morel-Lavallée lesion. J Trauma 1997;42:1046-51.

2. Parra JA, Fernandez MA, Encinas B, Rico M. Morel-Lavallée effusions in the thigh. Skeletal Radiol 1997;26:239-41.

3. Claassen L, Franssen MA, de Loos ER. A rare case of hemorrhagic shock: Morel-Lavallée lesion. Clin Pract Cases Emerg Med 2019;3:417-20.
4. Hudson DA, Knottenbelt JD, Krige JE. Closed degloving injuries: results following conservative surgery. Plast Reconstr Surg 1992;89:853-5.

5. Diviti S, Gupta N, Hooda K, Sharma K, Lo L. Morel-Lavallee lesions-review of pathophysiology, clinical findings, imaging findings and management. J Clin Diagn Res 2017;11:TE01-4.

6. Opdam KTM, Coevering R van de, Haven I van der. Morel-Lavallée lesion: a not so rare, but often missed diagnosis. Open J Trauma 2017;1:64-5.

7. Zhang BF, Cong YX, Wang PF, Huang H, Wang H, Zhuang Y. Deep femoral artery branch pseudoaneurysm formation and injury after hip fracture surgery: a case series and a literature review. Medicine (Baltimore) 2018;97:e9872.

8. Potenza V, Saputo U, Catellani F, Farsetti P, Caterini R. Laceration of a branch of the profunda femoris artery caused by a spike of the displaced lesser trochanter in an inter-trochanteric femoral fracture. A case report. Int J Surg Case Rep 2016;24:1958.

9. Mueller DK, Greenberg JJ, Marshall WJ, Maull KI. Rupture of the deep femoral artery from blunt trauma. J Trauma 1995;39:1010-1.

10. Littlejohn L, Bennett BL, Drew B. Application of current hemorrhage control techniques for backcountry care: part two, hemostatic dressings and other adjuncts. Wilderness Environ Med 2015;26:246-54.

11. Nooh A, Wang CK, AlAseem A, Harvey EJ, Bernstein M. Acute thigh compartment syndrome due to an occult arterial injury following a blunt trauma: a case report. JBJS Case Connect 2020;10:e0506.

12. Burghardt RD, Siebenlist S, Döbele S, Lucke M, Stöckle U. Compartment syndrome of the thigh. A case report with delayed onset after stable pelvic ring fracture and chronic anticoagulation therapy. BMC Geriatr 2010;10:51.

13. Chen L, Reisner AT, Gribok A, Reifman J. Exploration of prehospital vital sign trends for the prediction of trauma outcomes. J Spec Oper Med 2010;10:55-62.

14. Singh R, Rymer B, Youssef B, Lim J. The Morel-Lavallée lesion and its management: a review of the literature. J Orthop 2018;15:917-21.

15. Nickerson TP, Zielinski MD, Jenkins DH, Schiller HJ. The Mayo Clinic experience with Morel-Lavallée lesions: establishment of a practice management guideline. J Trauma Acute Care Surg 2014;76:493-7. 